\title{
La Relation Banque-Entreprise Au Cameroun : Exploration Empirique Des Fondements
}

\author{
Joachim Christel Mbida, \\ Docteur en Sciences de Gestion \\ Assistant à $1^{\prime}$ Université de Yaoundé II, Cameroun
}

Doi:10.19044/esj.2020.v16n19p294 URL:http://dx.doi.org/10.19044/esj.2020.v16n19p294

\section{Résumé}

L'on assiste au cours des dernières décennies à de grandes mutations socio-économiques qui soulèvent d'importants défis épistémologiques en l'occurrence la question de la collaboration des banques avec les entreprises en quête de financement. L'ambition de la présente recherche est de contribuer à l'intelligence des facteurs de l'engagement des banques et des entreprises dans des relations financières. Pour ce faire, les théories des conventions et de la dépendance envers les ressources sont mobilisées. Cette approche s'inscrit dans une perspective dyadique. Le matériau empirique est constitué des données issues à la fois des banques et des entreprises. Les résultats suggèrent principalement que de quelque côté qu'on se trouve l'engagement des acteurs repose sur des facteurs à la fois objectifs et subjectifs.

Mots-clés: Relation-Banque-Entreprise-Fondement 


\title{
The Bank-Company Relationship in Cameroon: Empirical Foundation Exploration
}

\author{
Joachim Christel Mbida, \\ Docteur en Sciences de Gestion \\ Assistant à $1^{`}$ Université de Yaoundé II, Cameroun
}

\begin{abstract}
Over the past few decades, we have witnessed major socio-economic changes which raise important epistemological challenges, in this case the question of the collaboration of banks with companies seeking financing. The aim of this research is to contribute to the understanding of the factors involved in the engagement of banks and businesses in financial relationships. To do this, the theories mobilized are: theories of conventions, of dependence on resources. Our approach is part of a dyadic perspective. The empirical material is made up of data from both banks and companies. The results mainly suggest that wherever we are, stakeholder engagement is based on both objective and subjective factors.
\end{abstract}

Keywords: Relationship-Bank-Company-Foundation

\section{Introduction}

Dans le milieu des analystes géostratégiques, on assiste à un consensus sur les potentialités économiques de l'Afrique qui demandent néanmoins à être transformées en valeurs concrètes. Dans ce contexte caractérisé par la rareté des marchés boursiers (Fal, 2015), l'on s'interroge sur la responsabilité des banques à accompagner les États par des politiques financières conséquentes vis-à-vis des entreprises. Dans ce sens, il ressort qu'au cours de la période 2018, les banques camerounaises ont accordé 3372,11 milliards de francs CFA de crédits aux entreprises (BEAC, 2018). Au vu de l'importance socioéconomique des entreprises, ce taux est relativement faible car leur action d'entreprendre renvoie au besoin de financement. Ainsi il est nécessaire pour chaque entreprise de trouver des ressources financières adéquates pour son développement (Paturel, 2007). À travers la théorie des alliances, Garette et Dussauge (1995) soulignent qu'une forme de rapprochement entre ces deux acteurs est nécessaire mais de manière à ce que leur indépendance reste maintenue. En effet, les acteurs partenaires s'associent pour poursuivre des objectifs communs tout en conservant des intérêts qui leur sont propres. 
Cependant, cette quête de financement des entreprises constitue un véritable «casse-tête » chinois c'est-à-dire un problème très complexe qui fatigue $1^{\prime}$ esprit. En effet, les crédits accordés par les banques commerciales au Cameroun représentent $73,62 \%$ pour les grandes entreprises et $14,54 \%$ pour les petites structures et les 11,84\% pour les particuliers (BEAC, 2018). Pourtant celles-ci sont une source indispensable de croissance économique dans les économies émergentes (Douzounet et Yogo, 2012). Nonobstant leur importance socio-économique, elles font face à des difficultés de financement. Par ailleurs, une justification de cette situation indique que les pratiques et systèmes de gestion des entreprises sont parfois rudimentaires et ne satisfont pas les exigences des établissements bancaires (Schwarz, 2011). Ainsi cette opacité dans le fonctionnement amène les banques à adopter un comportement prudent. Cependant, la théorie de la collaboration de Podolny et Page (1998) relève que les deux parties doivent être guidées par le sens des obligations issues de la recherche de la conciliation des intérêts divergents.

En outre, les résultats des travaux de Rédis et Paré (2009) indiquent que les acteurs présentent de réelles faiblesses par rapport aux exigences que l'un ou l'autre formule. Autrement dit, le rapport banque-entreprise se caractérise par l'asymétrie d'information évoquée dans la théorie financière. $\mathrm{Au}$ stade actuel du débat académique spécialisé, certaines réflexions conduisent à s'interroger aussi sur la volonté des entreprises elles-mêmes qu'elles soient de grandes ou de petites tailles de nouer des relations financières avec les banques compte tenue des mutations relevées (concurrence, renforcement de la réglementation etc.). La pertinence conceptuelle de cette question trouve une justification dans l'idée émise par Rivaud-Danset (1993) selon laquelle la relation banque-entreprise est une convention qui pour être établie exige l'adhésion de chacune des parties.

Il devient judicieux de rechercher les fondements de la relation qui leur garantisse un meilleur accompagnement. Dans ce sens, Gardes et Maque (2012) relèvent les motifs qui fondent l'engagement des entreprises : le souci de payer des faibles tarifications ensuite l'accessibilité permanente au crédit enfin l'aspiration à supporter les garanties exigées. Pour les institutions financières, ils relèvent entre autres la bancabilité du projet et les risques liés à la personnalité des entrepreneurs. Au regard de tout ce qui précède, on ne saurait considérer le rapport banque-entreprise sans ressortir les facteurs sur lesquels se fondent le banquier et le dirigeant pour nouer une relation financière dans une action collective.

Ceci étant, la littérature dans une certaine mesure met en évidence les facteurs objectifs. Du point de vue de l'entreprise on peut relever particulièrement le coût du crédit et les garanties exigées. S'agissant des banques, elles mettent en exergue les informations financières et les documents comptables qui en résultent. Dans le contexte des pays en voie de 
développement notamment au Cameroun des travaux de Djoutsa Wamba et al. (2017) soulignent le caractère opaque et aléatoire de ces déterminants objectifs. En outre, en complément de ces données objectives jugées souvent insuffisantes pour une bonne prise de décision, Foliard (2010) s'intéresse aux facteurs subjectifs liés au profil du dirigeant et au comportement du banquier. Dans la même veine, d'autres chercheurs évoquent la réputation et l'image de marque (Cieply et Grondin, 2000; Maurel, 2012).

Les recherches menées sur le processus décisionnel ne s'attardent pas à l'étude de ces acteurs dans le cadre simultané. Par ailleurs, cette étude met en lumière les déterminants suivants: l'appartenance, la perception et le comportement. Toutes ces observations contribuent à justifier l'originalité de cette étude à partir de son angle d'analyse (sans marché financier). Tel est la problématique de la présente recherche qui peut être résumée par la question qui suit: Qu'est ce qui détermine l'engagement de la banque et de l'entreprise dans une relation financière? L'objectif de cet article est de contribuer à une meilleure compréhension des fondements du rapport des banques avec les entreprises non financières. Cette recherche s'organise autour de trois parties. La première présente le cadre d'analyse théorique. La deuxième est consacrée à la méthodologie et proposition de recherche et enfin la discussion des principaux résultats obtenus. Elle précède la portée managériale et scientifique de cette étude en guise de conclusion.

\section{Cadre d'analyse théorique}

L'étude du rapport banque-entreprise remonte aux années 1950. Roosa (1951) relève que le financement est un moyen d'assurer à l'entreprise sa croissance grâce au développement d'un savoir des banques de nature subjective. Par ailleurs, la forte asymétrie d'information existante entre la banque et l'entreprise dans le contexte des pays en voie de développement ne permet pas de tirer profit ou d'apprécier ce rapport. Dans cette partie, les asymétries d'information qui sous-tendent la relation banque-entreprise sont mises en exergue puis les facteurs qui traduisent l'engagement des acteurs sont relevés enfin le rapport banque-entreprise à la lumière du foisonnement théorique est abordé.

\subsection{Le rapport banque-entreprise : une explication par l'asymétrie d'information}

La théorie financière montre que le rapport des banques avec les entreprises conduit ces établissements financiers à bénéficier d'un pouvoir de contrôle sur les politiques financières de celles-ci. Barton et Gordon (1987) remettent en cause cet opportunisme des banques. Par ailleurs, les fonds des entreprises se présentent comme leur principale source de financement. Mais pour leur développement, ces fonds mobilisés s'avèrent le plus souvent 
insuffisants pour assurer cette croissance. Dans ce sens, le dirigeant d'entreprise est donc contraint à se tourner vers les banques pour rechercher les capitaux nécessaires pour le développement de son entreprise.

Toutefois, cette sollicitation du dirigeant est fonction de la capacité des banques à assurer leur développement. D’après Schwarz (2011), le risque inhérent qui caractérise cette relation banque-entreprise montre que l'accès au crédit bancaire n'est pas chose aisé pour les entreprises. Selon la théorie financière, les difficultés de celles-ci proviennent de l'exigence bancaire sur la quantification du risque en fonction de la nature de l'activité de l'entreprise.

En outre, ces acteurs mettent aussi en évidence des facteurs subjectifs (méfiance, incompréhension) pouvant justifier la frilosité de leurs rapports.

Stiglitz et Weiss (1981) relèvent qu'il existe deux sources d'asymétrie d'information qui limitent les rapports entre les deux parties. Ces auteurs relèvent d'une part la sélection adverse et d'autre part l'aléa moral traduisant la difficulté pour les acteurs à discriminer efficacement le projet à financer et les agissements parfois opportunistes. De ce fait, ces deux risques majeurs créent un climat de méfiance et d'incompréhension dans la relation banqueentreprise. Dans ce contexte d'incertitude, MacNeil (1978) préconise les approches relationnelles. En effet, elles sont caractérisées par une communication relativement dense et extensive des deux acteurs (Gardes et Machat, 2012) motivant leur envie à s'engager dans une relation financière. En outre, le «win-win » relationship des acteurs remplace l'affrontement exclusivement compétitif et conflictuel fondé sur leur choix opportunistes (N'Goala, 1998).

Ceci étant, la relation banque-entreprise devrait tenir sur la base de la prise en compte des facteurs pouvant permettre de réduire l'asymétrie d'information ou l'incertitude existante entre les deux acteurs les empêchant de fournir des informations fiables (Cieply et Grondin, 2000). Ainsi, il est souhaitable d'identifier les différents déterminants mis en évidence par les deux parties pour s'engager dans une relation financière.

\subsection{Les déterminants théoriques de l'engagement de la banque et de l'entreprise}

Le foisonnement théorique de cette étude s'appuie sur une synthèse des travaux empiriques sur les déterminants de la relation banque-entreprise dans un contexte de manque d'informations fiables. En effet, la présence de l'incertitude et de l'asymétrie d'information entre la banque et l'entreprise empêchent ces deux acteurs économiques à nouer une relation financière. Les résultats de Godham et al. (2007) ont mis en relief l'analyse du dossier de crédit du client dans le processus de financement.

Par ailleurs, les travaux de Bruns et Fletcher (2008) mettent en avant la performance financière et la structure actionnariale de l'entreprise dans la 
prise de décision des banquiers. Dans la même veine, Quiquerez et Crevoisier (2006) soulignent que l'appartenance clanique peut également constituer un atout dans la prise de décision des banquiers. En effet, le relationnel établi entre les acteurs génère de la confiance qui apparaît comme un facteur lié à l'éthique. Cette notion intervient en amont de la décision et a pour ambition de distinguer la bonne et la mauvaise façon d'agir de chaque partie (Mercier, 2002).

Parmi les facteurs de l'engagement des dirigeants dans l'envie de nouer une relation avec la banque, il faut noter les conditions de prêt (le taux d'intérêt, les garanties demandées, etc.). Dans le même sillon des idées, Mayer et al, (1995) soulignent que pour que la banque inspire pleinement confiance et que l'entreprise s'engage avec elle plusieurs critères doivent être réunis (compétence, capacité et bienveillance). Pendant des entretiens sur le terrain, un dirigeant d'entreprise déclarait «Un chargé de clientèle bienveillant ne se limite pas formellement au prêt qu'il est censé accorder mais il prend sans cesse des initiatives pour améliorer son offre par les conseils ». Ring et Van de Ven (1994) montrent que l'on ne peut comprendre le fonctionnement du rapport des banques avec les entreprises qu'en se référant à une double logique : l'exigence d'efficacité et le besoin d'équité.

\subsection{Quelques grilles de lecture mobilisables}

La relation banque-entreprise apparait comme un « nœud de contrats » entre les acteurs selon la théorie de l'agence. Dans ce sens, Jensen et Meckling (1976) considèrent ces organisations comme des fictions légales servant pour un ensemble de relations contractuelles ayant des intérêts distincts. Ainsi, ces auteurs insistent sur la connaissance «pertinente » dans la prise de décision que les acteurs doivent être capables d'opérer dans leur choix.

Le rapport des banques avec les entreprises non financières s'inscrivant dans une perspective inter-organisationnelle repose sur des questions d'asymétrie d'information et du risque de crédit caractérisant la relation financière (Stiglitz et Weiss, 1981). Les capacités et les connaissances que possèdent les acteurs sont parfois limitées. Cette limite conceptualisée à l'origine par H. Simon a pour conséquence la difficulté d'établir des liens entre les parties. Dans ce sens, les asymétries d'information entre les acteurs sont au cœur de l'analyse du choix de financement.

Par ailleurs, cette relation doit s'apprécier bien au-delà de la théorie financière pour approfondir les échanges entre les deux parties. De ce fait, la théorie des relations humaines porte une attention particulière sur les comportements des acteurs qui modèlent l'engagement. Il s'agit ici de dynamiser les attitudes grâce à une nouvelle conception des rapports humains qui favorisent donc les liens sociaux mais aussi la négociation entre les acteurs (Friedberg, 1983). Lehman et Neuberger (2001) relèvent que les entraves à 
l'opportunisme des deux parties peuvent être sensiblement réduites par un climat de confiance (honnêteté et intégrité) entre les deux. De plus leur capacité à éclairer et à se donner des informations fiables afin de s'aider à prendre conscience et se pousser à l'action. Lorsque l'interaction devient un enjeu important et spécifique la confiance ne constitue plus une condition suffisante pour l'envie des acteurs à s'engager (Granovetter, 1992).

C'est à travers le comportement et le professionnalisme des deux acteurs que ceux-ci peuvent fonder leur engagement. Dans ce sens, pendant l'échange, le banquier doit s'adapter à l'entrepreneur sur certains points notamment « la vitesse de la voix » afin de le mettre en confiance. Cette approche favorise ainsi le relationnel (MacNeil, 1978) et soutient une meilleure socialisation des acteurs.

Dans les rapports complexes, la théorie des ressources permet une analyse stratégique des organisations et semble répondre aux raisons de leur succès ou de leur échec. Cette théorie de Pfeffer et Salancik (1978) aborde l'engagement sous l'angle de la "nécessité » pour les acteurs de trouver leur intérêt à la relation. Dès lors, il paraît essentiel de comprendre comment les deux parties intègrent leur processus décisionnel.

\section{Cadre méthodologie et formulation de la proposition de recherche}

Dans cette partie, il est opportun d'insister sur deux points majeurs donc le premier est relatif à la collecte et l'analyse d'information (2.1). Le second à la discussion des résultats obtenus (2.2) et la portée de la recherche est exposée en conclusion. Pour répondre à la problématique, les données qualitatives sont mobilisées pour comprendre de façon fine les processus mis en œuvre aux niveaux individuels (Singh et al, 2015). Une posture de type interprétativiste dans le souci de cohérence avec la question de recherche vise à comprendre plutôt qu'à mesurer ou à tester (Aktouf, 2008). De plus avec la complexité et la contingence de l'objet de la communication (D'Iribarne, 2004) l'approche mobilisée est dyadique dans la mesure où, l'attention est portée aux deux acteurs.

\section{1. Échantillon, collecte et analyse des données}

L'induction est basée sur une étude de cas multiples (Yin, 1994). L'article porte sur quatre banques (filiales occidentales, panafricaine et banque domestique) et sur un ensemble de trois entreprises à savoir : les Très Petites Entreprises (TPE), les Petites et Moyennes Entreprises (PME) et les Grandes Entreprises (GE) ayant sollicité le financement auprès des banques de cette recherche. Toutefois, ces acteurs ont souhaité conserver l'anonymat. L'outil de collecte des données était l'entretien semi directif qui s'inscrit dans une bonne scientificité. 20 chargés de clientèles et 30 gestionnaires d'entreprises ont été interrogés. Ces personnes ressources se répartissent entre Yaoundé et 
Douala deux villes attractives du Cameroun. Le tableau 1 ci-dessous donne un aperçu des guides d'entretien utilisés.

Tableau 1 : Guide d'entretien des deux acteurs

\begin{tabular}{|c|c|c|}
\hline \multirow{2}{*}{\multicolumn{3}{|c|}{ ENTREPRISES }} \\
\hline & & \\
\hline Thématiques & Libéllés & Objectifs \\
\hline $\begin{array}{c}\text { Thématique } \\
1\end{array}$ & $\begin{array}{l}\text { Les critères d'acceptation des } \\
\text { offres de produits financiers }\end{array}$ & $\begin{array}{l}\text { Ressortir les éléments qui fondent leur } \\
\text { engagement avec la banque (les facteurs } \\
\text { objectifs et subjectifs) }\end{array}$ \\
\hline $\begin{array}{l}\text { Thématique } \\
2\end{array}$ & $\begin{array}{l}\text { Les variables permettant de } \\
\text { percevoir le chargé de clientèle }\end{array}$ & $\begin{array}{l}\text { Le comportement du banquier recherché } \\
\text { (l'expérience, la qualité, les attitudes) }\end{array}$ \\
\hline $\begin{array}{l}\text { Thématique } \\
\mathbf{3}\end{array}$ & $\begin{array}{l}\text { L'origine de la pérennité ou de } \\
\text { la rupture de la relation }\end{array}$ & Ressortir les critères \\
\hline \multicolumn{3}{|c|}{ BANQUES } \\
\hline Thématiques & Libéllés & Objectifs \\
\hline $\begin{array}{c}\text { Thématique } \\
1\end{array}$ & $\begin{array}{l}\text { Les motifs qui fondent la } \\
\text { décision de financement }\end{array}$ & $\begin{array}{c}\text { Ressortir les facteurs (objectifs et } \\
\text { subjectifs) qui permettent à la banque de } \\
\text { nouer une relation financière }\end{array}$ \\
\hline $\begin{array}{l}\text { Thématique } \\
2\end{array}$ & Le profil des dirigeants & $\begin{array}{l}\text { Le comportement des chefs } \\
\text { d'entreprises recherché (l'expérience, la } \\
\text { qualité, les attitudes) }\end{array}$ \\
\hline $\begin{array}{c}\text { Thématique } \\
\mathbf{3}\end{array}$ & La perception des banquiers. & $\begin{array}{c}\text { Le ressenti des chargés de clientèle vis à } \\
\text { vis des entrepreneurs }\end{array}$ \\
\hline
\end{tabular}

\section{Source: Auteur}

Les données collectées sont saisies à l'aide du logiciel CSPRO 6.0. Une analyse de contenu thématique permet de comprendre les acteurs dans leur contexte (Mckeever, Jack et Anderson, 2015). Le logiciel Sphinx Quali a été utilisé pour le traitement des données. Environ 105 heures ont été nécessaires pour retranscrire les entretiens sous format Word avec saisie de 150615 mots afin de constituer des catégories et des verbatim qui facilitent l'analyse. Cette attitude interprétativiste a conduit à émettre une proposition de recherche en s'inspirant des études antérieures (Burgelman, 1983; Johnson et al., 2003 ; Jarzabkowski, 2007, etc.). Ainsi, une proposition centrale se dégage: les acteurs fondent leur engagement sur un certain nombre de déterminants tant objectifs que subjectifs. Plus spécifiquement il s'agit :

Sous proposition 1: d'identifier les paramètres objectifs et subjectifs sur lesquels s'appuie l'entreprise pour nouer une relation avec la banque.

Sous proposition 2 : d'identifier les éléments sur lesquels se fonde la banque pour s'engager avec l'entreprise.

\subsection{Exposition et discussion des résultats}

Les résultats sont exposés ainsi que leur description statistique. De ce fait, cette discussion des résultats s'appuie sur une synthèse de la littérature 
empirique. En effet, elle ressort les déterminants visant à mieux comprendre ce que les deux acteurs mettent en exergue pour leurs rapports. Par la suite, la proposition émise peut être confirmée ou infirmée afin d'aider la banque et l'entreprise dans leur collaboration.

\subsubsection{Les liaisons entre les facteurs et l'engagement des entreprises}

Le graphique ci-après présente une pyramide des facteurs objectifs ainsi que les statistiques obtenues à partir de l'analyse des données collectées.

Graphique 1 : Le taux de considération des facteurs objectifs

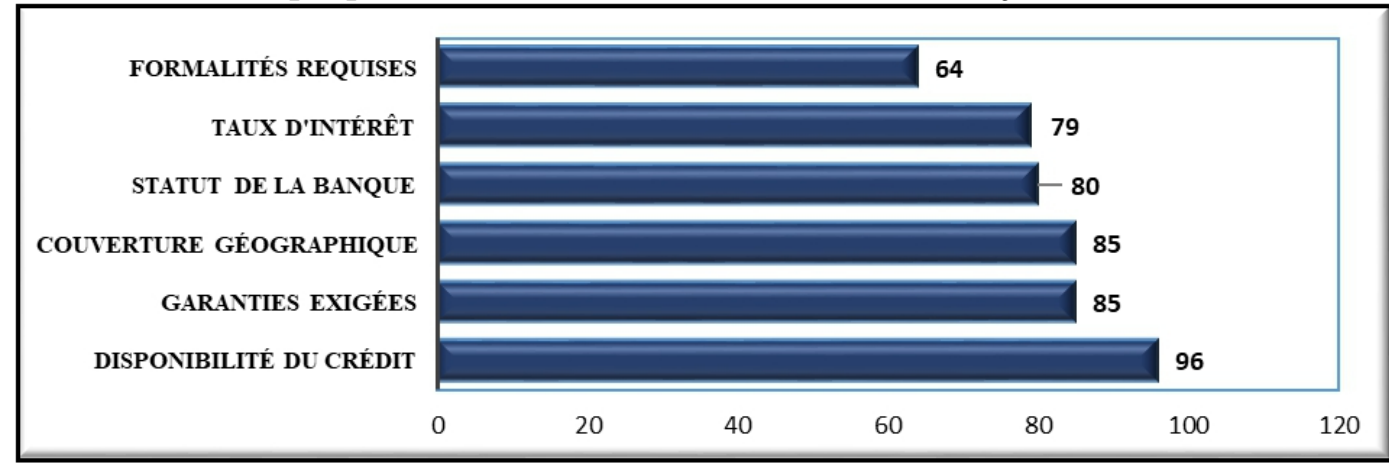

Source: Auteur

L'analyse descriptive sur le taux de considération des facteurs objectifs indique que la disponibilité du crédit bancaire est le facteur le plus représenté avec 96\% d'occurrences. Mais les travaux de Petersen et Rajan (1994) révèlent que la durée de la relation banque-entreprise a une incidence significative sur ce facteur. Ce déterminant (disponibilité du crédit) montre que l'envie de s'engager pour les entreprises part du présupposé que celles-ci sont en quête de financement. Compte tenu de cette spécificité, Pfeffer et Salancik (1978) soulignent que les entreprises ne sont pas pour la plupart autonomes en termes de capital mais dépendantes des banques (la théorie de la dépendance des ressources).

Tableau 2 : Le taux de considération des variables objectives

\begin{tabular}{|c|c|c|c|c|}
\hline \multirow{2}{*}{ Variables objectives } & \multicolumn{3}{|c|}{ Type d'entreprise } & $\begin{array}{c}\text { Ensemble } \\
\text { (Moyenne } \\
\text { arithméthique } \\
\text { pondérée) }\end{array}$ \\
\cline { 2 - 5 } & GE & PME & TPE & \\
\hline Disponibilité du crédit (DC) & 100 & 94 & 100 & 96 \\
\hline Garanties exigées (GE) & 40 & 91 & 80 & 85 \\
\hline $\begin{array}{c}\text { Couverture géographique } \\
\text { (CG) }\end{array}$ & 80 & 86 & 85 & 85 \\
\hline Statut de la banque (SB) & 80 & 84 & 70 & 80 \\
\hline Taux d'intérêt (TI) & 70 & 88 & 60 & 79 \\
\hline Formalités requises & 90 & 76 & 28 & 64 \\
\hline Indice objectif & $\mathbf{7 7}$ & $\mathbf{8 7}$ & $\mathbf{7 0}$ & $\mathbf{8 2}$ \\
\hline
\end{tabular}


Les valeurs sont en \%

Source: Auteur

Légendes: GE: Grandes Entreprises; PME: Petites et Moyennes Entreprises; TPE: Très Petites Entreprises.

Un constat est fait de tel sorte que quel que soit le type d'entreprise la disponibilité du crédit est le facteur le plus prépondérant. L'Indice Objectif Global (IOB) est une moyenne arithmétique pondérée des pourcentages réponses positives des six facteurs objectifs dans l'établissement des rapports banque/entreprise. Sa formule est définie par :

$$
\mathbf{I O B}_{i}=\frac{\sum_{k=1}^{6} \boldsymbol{w}_{k} \boldsymbol{F}_{k i}}{\sum_{k=1}^{6} \boldsymbol{w}_{k}} ; \text { avec }\left\{\begin{array}{l}
F_{k i}=\left\{\begin{array}{l}
1, \text { sil'entrepriseiconsidèrele facteurk comme un déter minant } \\
0, \text { sin on }
\end{array}\right. \\
0 \leq w_{k} \leq 1, \text { le poids du facteurk }
\end{array}\right.
$$

L'indice d'objectivité est évalué à $82 \%$ dans l'ensemble. Cet indice est plus important dans les PME (87\%) ensuite dans les GE (77\%) et enfin 70\% pour les TPE. Pour ces dernières, Tioumagneng (2012) insinue qu'elles privilégient le financement informel (tontines) et choisissent parfois le financement classique en fonction de leur capacité à satisfaire certains besoins. La réticence de ces petites entreprises amène les banquiers à revoir leur comportement relationnel (l'approche marketing de MacNeil, 1978). Le lien entre facteurs subjectifs et l'engagement des entreprises est illustré sur la figure 2 ci-dessous.

Graphique 2 : Le taux de considération des facteurs subjectifs

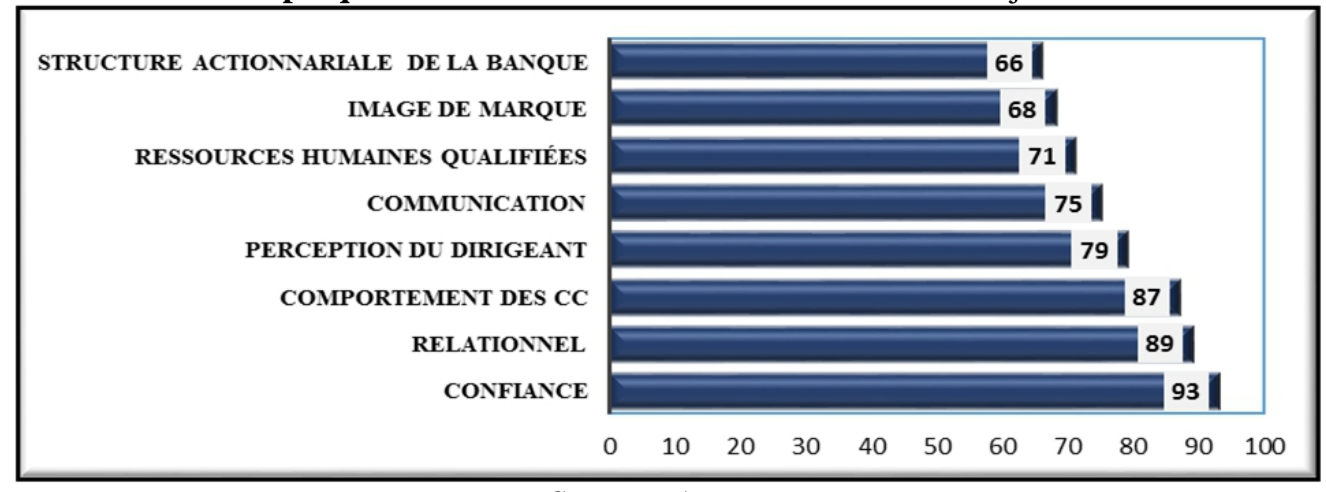

Source : Auteur

D'après le graphique ci-dessus, on remarque que les déterminants sont alignés par ordre de représentativité croissante. Ainsi, la confiance est le facteur le plus représenté avec 93\%. L'étude de Lamarque (2002) souligne qu'il est préférable de mieux appréhender les aspects humains dans les rapports car la méfiance aboutit souvent à des incompatibilités entre les deux acteurs. En 
outre, les résultats de Maurel (2012) mettent en évidence l'image de marque et la communication comme facilitant l'engagement des entreprises. Partant des observations des dirigeants interviewés, le tableau ci-après ressort certains comportements :

Tableau 3 : La synthèse des comportements des chargés de clientèle

\begin{tabular}{|c|l|}
\hline $\begin{array}{c}\text { Comportement } \\
\begin{array}{c}\text { Chargés de clientèle } \\
\text { courtois ou } \\
\text { accueillants }\end{array}\end{array}$ & $\begin{array}{l}\text { Ces banquiers font bonne impression au moment qu'ils reçoivent } \\
\text { (serviable, plaisant, toujours accessible, souriant...). }\end{array}$ \\
\hline $\begin{array}{c}\text { Chargés de clientèle } \\
\text { réticents }\end{array}$ & $\begin{array}{l}\text { Ils ne manifestent pas ouvertement leur pensée pendant l'échange. } \\
\text { Ils sont réservés, ne voulant pas dire tout ce qu'ils savent, hésitants } \\
\text { et n'éprouvent aucune empathie pour leur interlocuteur. }\end{array}$ \\
\hline $\begin{array}{c}\text { Chargés de clientèle } \\
\text { arrivistes }\end{array}$ & $\begin{array}{l}\text { Ceux-ci sont des parvenus, prétentieux et imbus de leur personne. } \\
\text { Pendant l'échange, ils passent plus de temps à s'occuper d'autres } \\
\text { choses (ordinateurs, manipuler le téléphone, etc.) que de son vis-à- } \\
\text { vis. }\end{array}$ \\
\hline $\begin{array}{c}\text { Chargés de clientèle } \\
\text { novices }\end{array}$ & $\begin{array}{l}\text { Ceux-ci étant débutants dans ce domaine n'ont pas une expérience } \\
\text { en la matière. Leurs entretiens sont laborieux, très balbutiants. }\end{array}$ \\
\hline $\begin{array}{c}\text { Chargés de clientèle } \\
\text { professionnels }\end{array}$ & $\begin{array}{l}\text { Ils aident les dirigeants à atteindre leurs objectifs au cours de } \\
\text { l'échange. Ils font preuve d'une grande écoute pour trouver des } \\
\text { stratégies de développement des entreprises. }\end{array}$ \\
\hline
\end{tabular}

Source: Auteur

Au cours des entretiens sur le terrain, un banquier disait : «Pour influencer favorablement ses potentiels clients et jouir auprès d'eux d'une bonne impression, maitriser ses attitudes se révèle donc indispensable ». Il en ressort que l'existence de liens sociaux entre les acteurs tend à favoriser une meilleure collaboration.

Tableau 4 : Le taux de considération des variables subjectives

\begin{tabular}{|c|c|c|c|c|}
\hline \multirow[b]{2}{*}{ Variables subjectives } & \multicolumn{3}{|c|}{ Type d'entreprise } & \multirow[b]{2}{*}{$\begin{array}{c}\text { Ensemble } \\
\text { (Moyenne } \\
\text { arithméthique } \\
\text { pondérée) }\end{array}$} \\
\hline & GE & PME & TPE & \\
\hline Confiance $(\mathrm{CON})$ & 100 & 93 & 90 & 93 \\
\hline Relationnel (RELAT) & 80 & 96 & 75 & 89 \\
\hline $\begin{array}{c}\text { Comportement des Chargés de } \\
\text { clientèle (CCC) }\end{array}$ & 100 & 89 & 78 & 87 \\
\hline Perception du dirigeant (PD) & 30 & 82 & 83 & 79 \\
\hline Communication $(\mathrm{COM})$ & 70 & 78 & 70 & 75 \\
\hline Ressources humaines qualifiées (RHQ) & 80 & 62 & 90 & 71 \\
\hline Image de marque (IM) & 80 & 77 & 43 & 68 \\
\hline $\begin{array}{l}\text { Structure actionnariale de la banque } \\
\qquad(\mathrm{SAB})\end{array}$ & 80 & 62 & 73 & 66 \\
\hline Indice subjectif les valeurs sont en \% & 77 & 80 & 75 & 78 \\
\hline
\end{tabular}

Source: Auteur 
Ces facteurs relèvent de l'interpersonnel et sont à ce titre discutables. L'Indice Subjectif Global (ISB) comme l'IOB est une moyenne arithmétique pondérée des pourcentages des huit facteurs subjectifs dans l'établissement d'une relation entre une entreprise et une banque. Sa formule est ainsi définie par :

$$
\boldsymbol{I S B}_{i}=\frac{\sum_{k=1}^{8} v_{k} \boldsymbol{F}_{k i}}{\sum_{k=1}^{8} v_{k}} ; \text { avec }\left\{\begin{array}{l}
F_{k i}=\left\{\begin{array}{l}
1, \text { sil'entrepriseiconsidèrele facteurk comme un déter minant } \\
0, \text { sin on }
\end{array}\right. \\
0 \leq v_{k} \leq 1, \text { le poids du facteurk }
\end{array}\right.
$$

L'indice de subjectivité est évalué à $78 \%$ dans l'ensemble. Il est plus important dans les PME (80\%) ensuite dans les GE (77\%) et enfin 75\% pour les TPE. Ces résultats sous-tendent le principe central de la théorie de la contingence «Tout dépend de». Dans ce sens, Lawrence et Lorsch (1969) montrent que les facteurs subjectifs rendent complexes l'engagement des entreprises.

\subsubsection{L'engagement des banques : Une prépondérance des facteurs subjectifs sur les facteurs objectifs.}

À partir des observations du terrain, l'engagement est influencé par bon nombre de facteurs (cf. graphique 2 ci-après). Cette analyse présente par ordre de détermination décroissante les facteurs objectifs les plus pertinents à savoir : NA (95\%) et RISQ-RE-NG (85\%). Ce résultat corrobore celui de Reinke (1998) qui souligne que la nature de l'activité joue un rôle dans l'envie de nouer une relation.

Graphique 3: Le taux de détermination des déterminants objectifs

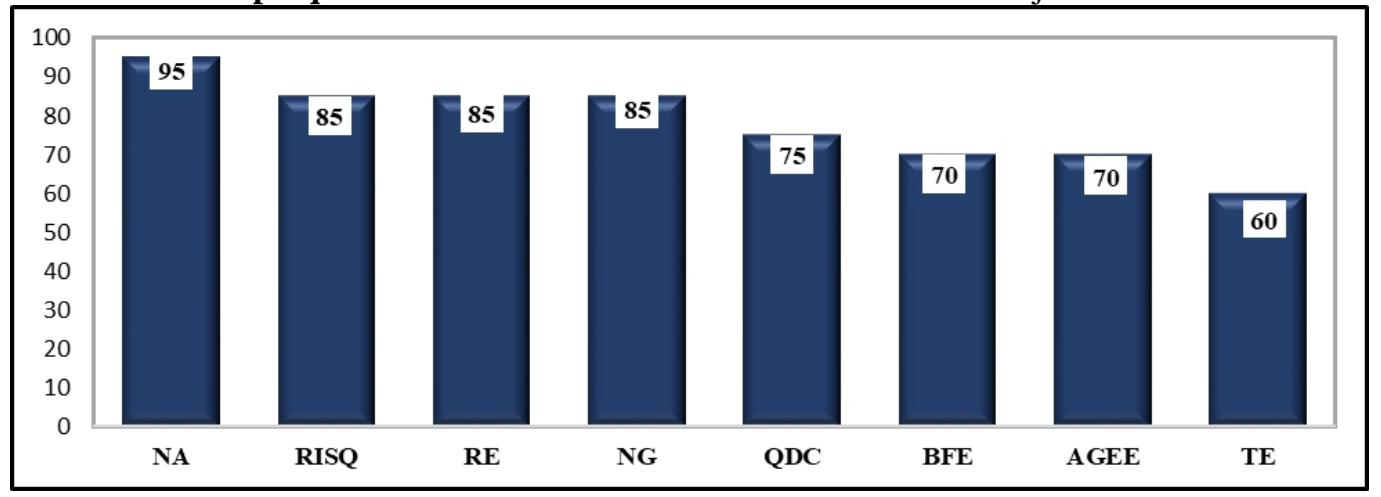

\section{Source: Auteur}

Légendes: NA: Nature de l'activité, RISQ: Risque, RE: Reputation de 1'Entreprise, NG: Niveau de Garantie, QDC: Qualité du Dossier du Crédit, BFE: Bilan Financier de l'Entreprise, AGEE: Age de l'entreprise et TE: Taille de l'entreprise.

Ces résultats sont à rapprocher des travaux de Foliard (2010) qui révèlent que le risque et la nature de l'activité sont deux raisons qui poussent la banque à s'engager. Lors de l'enquête de cette étude, un Chargé de clientèle avançait : «je valide un projet parce qu'il est solide techniquement et commercialement 
». Dans le même sillage des idées, Godham et al. (2007) soulignent que l'envie de s'engager comprend plusieurs phases dont la plus importante est la qualité du dossier de crédit. En outre, (Cieply et Grondin, 2000 ; Bruns et Fletcher, 2008) montrent que les banques cherchent à réduire l'asymétrie d'information par l'analyse de plusieurs facteurs dont la réputation et la performance financière. Les travaux d'Um-Ngouem et Ekonne (2013) indiquent que la garantie est l'un des facteurs pris en considération au même titre que l'âge et la taille de l'entreprise. Le tableau ci-dessous montre que l'indice d'objectivité est évalué à $78,1 \%$ dans l'ensemble. Les filiales de banques étrangères considèrent un peu plus les déterminants objectifs $(87,5 \%)$ pour recourir à un engagement.

Tableau 5: La relation entre déterminants objectifs selon les différentes banques

\begin{tabular}{|c|c|c|c|c|}
\hline $\begin{array}{c}\text { Type de } \\
\text { banque }\end{array}$ & $\begin{array}{c}\text { Domestiq } \\
\text { ue }\end{array}$ & $\begin{array}{c}\text { Filiales de } \\
\text { banques }\end{array}$ & $\begin{array}{c}\text { Filiale de banque } \\
\text { panafricaine }\end{array}$ & $\begin{array}{c}\text { Ensemble } \\
\text { (Moyenne arithméthique } \\
\text { pondéré) }\end{array}$ \\
\hline NA & 80,0 & 100,0 & 100,0 & 95,0 \\
\hline RISQ & 60,0 & 100,0 & 90,0 & 85,0 \\
\hline RE & 60,0 & 100,0 & 90,0 & 85,0 \\
\hline NG & 100,0 & 60,0 & 90,0 & 85,0 \\
\hline QDC & 40,0 & 100,0 & 80,0 & 75,0 \\
\hline BFE & 40,0 & 80,0 & 80,0 & 70,0 \\
\hline AGEE & 40,0 & 100,0 & 70,0 & 70,0 \\
\hline TE & 20,0 & 60,0 & 80,0 & 60,0 \\
\hline IOB & 55,0 & 87,5 & 85,0 & 78,1 \\
\hline
\end{tabular}

Source: Auteur

Légendes: NA: Nature de 1'activité, RISQ: Risque, RE: Reputation de 1'Entreprise, NG: Niveau de Garantie, QDC: Qualité du Dossier du Crédit, BFE: Bilan Financier de 1'Entreprise, AGEE: Age de l'entreprise et TE: Taille de l'entreprise, IOB: Indice d'Objectivité.

Lehmann et Neuberger (2001) montrent que l'engagement dans une relation financière s'inscrit dans une logique de complémentarité des déterminants. Le graphique ci-après présenté par ordre de détermination décroissante dénote que les facteurs subjectifs les plus significatifs sont NI (95\%) et BIENV-SIN (88,9\%). Selon Anderson et Narus (1990) la bienveillance et la sincérité pour les banquiers est la croyance selon laquelle le dirigeant est investi de bonnes intentions et qu'il manifeste de l'attachement à son égard. Pendant les enquêtes, un banquier affirmait «Bien connaître son client permet de mieux l'approcher et savoir quelle démarche est susceptible de lui apporter une plus grande valeur ajoutée ». 
Graphique 4 : Le taux de détermination des déterminants subjectifs

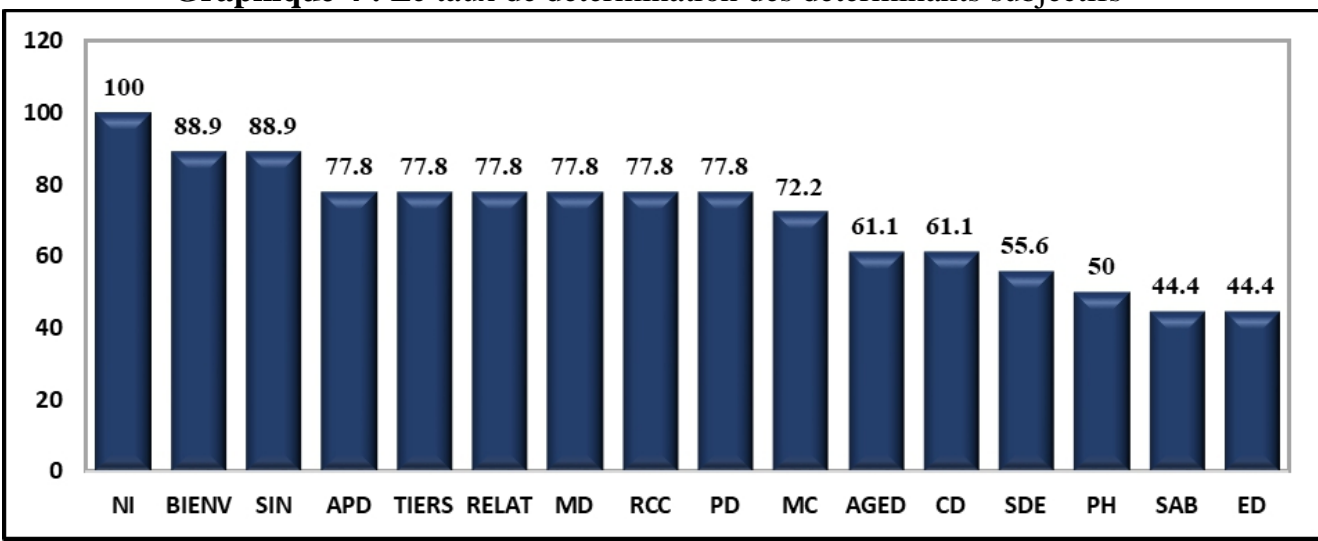

Source: Auteur.

Légendes: NI: Niveau d'information, BIENV: Bienveillance, SIN: Sincérité, APD: Appartenance du Dirigeant, TIERS: Tiers, RELAT: Relationnel, MD: Motivation du Dirigeant, RCC: Ressenti du Chargé de Clientèle, PD: Profil du Dirigeant, MC: Mouvement du compte, AGED: Age du Dirigeant, CD: Compétence du Dirigeant, SDE: Statut du Dirigeant d'Entreprise; PH: Pression de la Hierachie, SAB: Structure Actionnariale de la Banque, ED: Expérience du Dirigeant.

Dans le contexte des économies émergentes, les travaux de Djoutsa Wamba et al., (2017) ont relevé le caractère fantaisiste de la production des facteurs objectifs. C'est pourquoi en complément de ces données souvent opaques, les banquiers s'intéressent à des facteurs subjectifs. Dans ce sens, les travaux d'Um-Ngouem et Ekonne (2013) mettent en évidence le profil du dirigeant et l'âge du dirigeant. Pour ce dernier déterminant, Reinke (1998) souligne que les jeunes entrepreneurs sont souvent perçus comme des emprunteurs à haut risque. L'indice de subjectivité est évalué à $69,1 \%$ dans l'ensemble. Il est plus important dans les filiales des banques étrangères $(77,5 \%)$ ensuite dans les filiales de banques panafricaines $(69,4 \%)$ et enfin $60 \%$ pour les banques domestiques.

Tableau 6: Le taux de considération des variables de subjectivité selon le type de banques

\begin{tabular}{|l|l|l|l|l|}
\hline $\begin{array}{l}\text { Type de } \\
\text { banque }\end{array}$ & Domestique & $\begin{array}{l}\text { Filiale de } \\
\text { banque } \\
\text { étrangère }\end{array}$ & $\begin{array}{l}\text { Filiale de } \\
\text { banque } \\
\text { panafricaine }\end{array}$ & $\begin{array}{l}\text { Ensemble } \\
\text { (Moyenne } \\
\text { arithméthique } \\
\text { pondérée) }\end{array}$ \\
\hline NI & 80,0 & 100,0 & 100,0 & 95,0 \\
\hline APD & 100,0 & 60,0 & 80,0 & 80,0 \\
\hline TIERS & 100,0 & 60,0 & 80,0 & 80,0 \\
\hline BIENV & 40,0 & 100,0 & 90,0 & 80,0 \\
\hline SIN & 40,0 & 100,0 & 90,0 & 80,0 \\
\hline RELAT & 60,0 & 100,0 & 80,0 & 80,0 \\
\hline MD & 100,0 & 80,0 & 70,0 & 80,0 \\
\hline MC & 40,0 & 100,0 & 70,0 & 70,0 \\
\hline
\end{tabular}




\begin{tabular}{|l|l|l|l|l|}
\hline RCC & 40,0 & 100,0 & 70,0 & 70,0 \\
\hline PD & 60,0 & 80,0 & 70,0 & 70,0 \\
\hline AGED & 40,0 & 60,0 & 70,0 & 60,0 \\
\hline PH & 100,0 & 40,0 & 40,0 & 55,0 \\
\hline CD & 20,0 & 80,0 & 60,0 & 55,0 \\
\hline SDE & 20,0 & 80,0 & 50,0 & 50,0 \\
\hline SAB & 80,0 & 60,0 & 30,0 & 50,0 \\
\hline ED & 40,0 & 40,0 & 60,0 & 50,0 \\
\hline ISB & 60,0 & 77,5 & 69,4 & 69,1 \\
\hline
\end{tabular}

Source: Auteur

Légendes: NI: Niveau d'information, APD: Appartenance du Dirigeant, TIERS: Tiers, BIENV: Bienveillance, SIN: Sincérité, RELAT: Relationnel, MD: Motivation du Dirigeant, MC: Mouvement du compte, RCC: Ressenti du Chargé de Clientèle, PD: Profil du Dirigeant, , AGED: Age du Dirigeant, PH: Pression de la Hierachie, CD: Compétence du Dirigeant, SDE: Statut du Dirigeant d'Entreprise, SAB: Structure Actionnariale de la Banque, ED: Expérience du Dirigeant, ISB: Indice de Subjectivité.

Sur un échantillon de 20 chargés de clientèle plus de $80 \%$ retiennent la motivation comme facteur d'engagement dans une relation financière au même titre que les aspects liés au comportement (le regard, l'expression du visage, la voix) illustrés dans le tableau ci-après. Au cours des investigations, un chargé de clientèle déclarait "Un entrepreneur motivé est passionné, audacieux et avance, il ne se contente pas d'envisager ».

Tableau 7: La synthèse des Comportements du dirigeant

\begin{tabular}{|l|l|}
\hline \multicolumn{1}{|c|}{ Personnalités } & \multicolumn{1}{c|}{ Caractéristiques } \\
\hline Clients avérés & Ils sont rassurants et possèdent un esprit d'ouverture. \\
\hline $\begin{array}{l}\text { Clients beaux } \\
\text { parleurs }\end{array}$ & $\begin{array}{l}\text { Ils entrent en rapport de force avec le chargé de clientèle afin de } \\
\text { s'affirmer auprès de celui-ci. }\end{array}$ \\
\hline Clients arrivistes & $\begin{array}{l}\text { Les personnes voulant parvenir à leurs fins peu importent les moyens } \\
\text { utilisés. }\end{array}$ \\
\hline Clients débutants & Ils sont incertains, sans assurance aucune. \\
\hline Clients pressés & $\begin{array}{l}\text { Les personnes motivées par l'espoir d'entrer vite en possession de } \\
\text { l'argent. }\end{array}$ \\
\hline Clients ambitieux & $\begin{array}{l}\text { Les personnes qui ont foi à leur projet et se donnent les moyens pour } \\
\text { atteindre leur objectif. }\end{array}$ \\
\hline Clients rêveurs & $\begin{array}{l}\text { Les individus présentant des projets hors normes pour dire non } \\
\text { bancables. }\end{array}$ \\
\hline
\end{tabular}

Source: Auteur

$80 \%$ des chargés de clientèle mettent en lumière la théorie managériale de Barnard (1938) résidant dans l'idée qu'il est fondamental de prendre en considération les comportements réels des dirigeants durant l'entretien. Les tableaux ci-dessous présentent la synthèse des résultats de cette étude. D'après ces tableaux les facteurs les plus significatifs pour les deux acteurs sont en moyenne supérieurs à $70 \%$ et permettent de valider cette proposition de recherche. Grosso modo, ces résultats montrent que la probabilité que la 
banque et l'entreprise nouent une relation financière pourrait être expliquée en partie par la prise en compte de ces facteurs.

Tableau 8 : Synthèse des résultats des acteurs les plus importants $(\%>70)$

\begin{tabular}{|c|c|c|c|}
\hline \multicolumn{2}{|c|}{ ENTREPRISES } & \multicolumn{2}{c|}{ BANQUES } \\
\hline Facteurs objectifs & Facteurs subjectifs & Facteurs objectifs & Facteurs subjectifs \\
\hline DC & CON & NA & NI \\
GE & RELAT & RISQ & BIENV \\
CG & CCC & RE & SIN \\
SB & PD & NG & APD \\
TI & COM & BFE & TIERS \\
& RHQ & AGEE & RELAT \\
& & & MD \\
\hline
\end{tabular}

Source: Auteur

Au regard des développements précédents et du cadre théorique sous-jacent, les résultats de cette étude ressortent les déterminants suivants : comportement des chargés de clientèle, perception des deux acteurs, appartenance du dirigeant et motivation. Ainsi les facteurs subjectifs dans un contexte de pays en voie de développement priment plus pour les relations banque-entreprise.

\section{Conclusion}

La banque et l'entreprise forment l'armature de toutes les économies. Ces deux acteurs sont considérés comme les sources essentielles de croissance économique de tout pays. Il est donc souhaitable que ces dernières se mettent ensemble car aucun d'eux ne saurait vivre en autarcie. Différentes théories mobilisées montrent leur importance dans l'orientation stratégique de chaque acteur. Ce travail de recherche s'est proposé d'étudier la question touchant les rapports banque-entreprise dans une relation financière. Les principaux résultats montrent que la décision d'engagement des acteurs repose sur les facteurs significatifs à la fois objectifs et subjectifs. Ils confirment la proposition principale de recherche et les sous-propositions.

L'implication managériale de cette étude est une vision croisée des déterminées car les acteurs doivent être sensibles aux facteurs trouvés. Ces travaux apportent des connaissances aisément utilisables aux deux acteurs. Les banquiers doivent être ouverts à la discussion et limiter les interprétations hâtives pour donner confiance afin de développer leur portefeuille client. Les facteurs mis en exergue sont l'occasion d'attirer l'attention ou préparer des jeunes entrepreneurs qui se lancent souvent désarmés face aux banquiers. La portée d'un point de vue scientifique de cette étude souligne qu'il faut aller au-delà du modèle transactionnel fondé sur les conditions de prêt pour avoir une vision relationnelle qui met en évidence l'intelligence des acteurs. Cette approche renvoie à une dynamique des théories entre autres sociologique et psychosociologique qui doivent s'immobiliser dans les grilles de lecture en mettant en évidence les facteurs subjectifs trouvés dans ses résultats. 
Cette recherche se démarque également des travaux classiques qui ne s'intéressent qu'à un seul pôle. Elle s'inscrit dans une logique fédératrice qui veut comprendre le fonctionnement de chaque partie car la relation banqueentreprise est un phénomène complexe qui nécessite des réflexions générales. Toutefois, ces résultats sont propres aux pays en voie de développement avec un marché financier embryonnaire voire inexistant qui sont bien différents de ceux des pays développés où la majorité des entreprises font appel au marché boursier assez développé et où l'information comptable et financière est moins opaque.

\section{References:}

1. Anderson, J. C., \& Narus, J. A. (1990). A Model of Distributor Firm and Manufacturer Firm Working Parternership Journal of Marketing, vol. 54, January.

2. Barton, S., \& Gordon, P. J. (1988). Corporate Strategy: Useful perspective for Study of Capital Structure? The Academy of Management Review. vol.12, $\mathrm{n}^{\circ} 1,1987$.

3. Banque des États de l'Afrique Centrale (BEAC). (2018). Rapport Annuel de la Banque.

4. Bruns, V., \& Fletcher, M. (2008). Banks' risk assessment of Swedish SMEs, Venture Capital, Vol. 10, $\mathrm{n}^{\circ} 2$.

5. Des Garets, V. Paquerot, M., \& Sueur, I. (2009). L'approche relationnelle dans les banques. Mythe ou réalité ?. Revue Française de Gestion, $\mathrm{n}^{\circ} 161$.

6. Djoutsa Wamba, L., Braune E., Hikkerova, L., \& Sahut, J. M. (2017). Indebtedness of young companies: the effects on their survival, Entrepreneurship \& Regional Development, vol. 29, $\mathrm{n}^{\circ} 12$.

7. Douzounet, M., \& Yogo, T. (2012). Capital Social et Survie des Entreprises au Cameroun, Rapport de Recherche du FR-CIEA, ${ }^{\circ}$ 26/12, juin.

8. Fal, G. (2015). Réformes nécessaires à l'approfondissement des marchés boursiers africains, Banque et Finance en Afrique, Ed, (pp. 109-150). RB.

9. Friedberg, E. (1983). La dimension sociale de la qualité, Enjeux. $\mathrm{n}^{\circ}$ 33.

10. Foliard, S. (2010). L'envie de financer un créateur néophyte. Approche exploratoire du premier entretien avec un banquier. Revue française de gestion. vol. 205, $\mathrm{n}^{\circ} 6$.

11. Gardes, N., \& Maque, I. (2012). La compétence relationnelle : une réponse à l'opportunisme des relations banque-entreprise. Revue internationale des PME, vol.25, $\mathrm{n}^{\circ} 1$. 
12. Godham, Y., Gueyiej, P., \& Siala, M. K. (2007). La décision de crédit: procédure et comparaison de la performance des quatre modèles de prévision et d'insolvabilité. Revue des Sciences de Gestion : Direction et Gestion, vol. 42, n 224/225.

13. Jensen, M., \& Meckling, W. (1976). Theory of the Firm: Managerial Behavior Agency Cost and Ownership Structure. Journal of Financial Economics, vol. $3, n^{\circ} 4$.

14. Lamarque, E. (2002). Pour une approche organisationnelle de la relation banque entreprise. Revue Sciences de Gestion, $\mathrm{n}^{\circ} 34$.

15. Lehmann, E., \& Neuberger, D. (2001). Do lending relationship matter? Evidence from Bank survey data in Germany. Journal of Economic Behavior and Organization, vol. 45.

16. MacNeil, I. (1978). Contracts: adjustment of long-term economic relations under classical, neo-classical, and relational contract law. Northwestern University Law Review, vol. 72, n 6.

17. Maurel, C. (2012). Les indicateurs de performance financières sont-ils vraiment les principaux critères d'octroi de financement bancaire

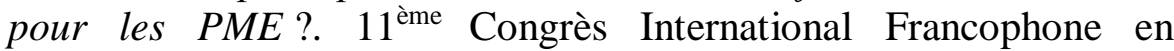
entrepreneuriat et PME. Lille, France.

18. Mayer, R. C., Davis, J. H., \& Schoorman, F. D. (1995) An Integrative Model of Organization Trust. Academy of Management Review, vol. $20, \mathrm{n}^{\circ} 3$.

19. Mbida, J. A. C. (2018). La relation des banques avec les entreprises non financières au Cameroun : à la recherche des fondements, thèse de Doctorat en Sciences de Gestion, Université de Yaoundé II.

20. Mckeever, E., Jack, S., \& Anderson, A. (2015). Embedded entrepreneurship in the creative reconstruction of place. Journal of Business Venturing, vol. 30, $\mathrm{n}^{\circ} 1$.

21. Mercier, S. (2002). Du discours à la réalité: théorie et pratique du management éthique . Cadres-CFDT, $\mathrm{n}^{\circ}$ 401-402, novembre.

22. N'goala, G. (1998). Epistémologie et théorie du marketing relationnel. Actes du $14^{\mathrm{ème}}$ Congrès de l'Association Française du Marketing, Bordeaux.

23. Pfeffer, J., \& Salancik, G. (1978). The external control of organizations: a resource dependence perspective. New York, Harper et Row.

24. Reinke, J. (1998). How to lend like mad and make a profit: A microcredit paradigm versus the start-up fund in South Africa. Journal of Development Studies, vol. 34, n³.

25. Ring, P. S., \& Van De Ven, A. H. (1994). Developmental Processes of Cooperative Interorganizational Relationships. Strategic Management Journal, vol. 19. 
26. Rivaud-Danset, D., \& Salais, R. (1993). Les conventions de financement. Premières approches théorique et empirique. Revue française d'économie, vol. $7, \mathrm{n}^{\circ} 4$.

27. Roosa, R., (1951). Interest Rates and the Central Bank. Money Trade and Economic. Growth, Macmillan, New York.

28. Quiquerez, F., \& O. Crevoisier, (2006). Financiarisation et Filialisation, quelle autonomie pour les PME?. 8ème Congrès International Francophone sur les PME, Fribourg, Suisse.

29. Schwarz, S. (2011). Les difficultés des Entrepreneurs pour acceder au crédit en République Démocratique du Congo, KfW Bankengruppe.

30. Singh, S. Corner, P., \& Pavlovich, K. (2015). Failed, not finished: A narrative approach to understanding venture failure stigmatization. Journal of Business Venturing, vol. 30, $\mathrm{n}^{\circ} 1$.

31. Stiglitz, J., \& Weiss, A. (1981). Credit Rationing in Market with Imperfect Information. The American Economic Review, vol. 71.

32. Tioumagneng, T. A., (2012). Banque et comportement d'endettement des entreprises. Recherches en Sciences de Gestion, vol. 2, no 89.

33. Um-Ngouem, M. T., \& Ekonne, E. A. (2013). Facteurs d'éligibilité des Très Petites Entreprises (TPE) au crédit des Etablissements de Microfinance (EMF) au Cameroun: une étude exploratoire. Communication aux $\mathrm{V}^{\text {ème }}$ journées internationales de la microfinance, Université d'Orléans.

34. Wamba, H. (2001). L'impact de l'asymétrie d'information dans l'optimisation de la valeur de l'entreprise : l'exemple de la PME camerounaise. SCSE Montréal. 